\title{
COMPENSATION OF INFLUENCE OF PROTECTOR COMPRESSION COEFFICIENTS IN TYRE INDUSTRY
}

UDC: 621.867 .2

Original scientific paper

https://doi.org/10.18485/aeletters.2019.4.1.5

\author{
Jasmina Radosavljevic ${ }^{1}$, Amelija Djordjevic ${ }^{1}$, Bojana M. Zlatkovic ${ }^{1}$, Biljana Samardzic ${ }^{2}$ \\ ${ }^{1}$ University of Nis, The Faculty of Occupational Safety, Nis, Serbia \\ ${ }^{2}$ University of Nis, The Faculty of Science and Mathematics, Nis, Serbia
}

\begin{abstract}
:
The system for protector cooling (protective rubber strip) in the tyre industry is presented in this paper. The analysis of compensation of protector compression coefficients influence on the protector length change between two neighbor transporters is done. Also, the compensation of protector compression coefficients influence on all transporters velocities is analyzed. Modelling and simulation of transportation system is performed in Matlab/Simulink.
\end{abstract}

\author{
ARTICLE HISTORY \\ Received: 25.01.2019. \\ Accepted: 30.03 .2019$. \\ Available: 31.03.2019.

\section{KEYWORDS} \\ Cascade systems, protector, \\ compensation, compression \\ coefficients, Matlab/Simulink
}

\section{INTRODUCTION}

Process of extrusion of protector - rubber strip, [1-5], is very important process in tyre industry. It is necessary to cool down the rubber strip to the room temperature, after the extrusion, in order to cut it into pieces of certain length depending on tyre dimension. The cascade transportation systems, Fig. 1, are used for the cooling [6]. These systems consist of 7 - 13 cascades and enable the protector cooling in the natural way without any additional tensions such that rubber strip relaxes on the transition from one transporter to another. However, in order to accomplish these requirements it is necessary to determine the velocity of each transporter and the whole line should have certain velocity, also. The velocities of the individual transporters are regulated on the basis of the measured tyre length between the transporters. The measuring is done by especially constructed measurer, and the information about the error is led to the thyristor controlled by DC motor controlling the next transporter.

The statical error of each cascade depends on the system input velocity and on the product of protector compression coefficients of the previous and the current cascade. Also, the inadequate choice of parameter values of the system can cause the cracking of rubber strip or of its extension, which leads to the system instability, chaos and in an extreme case to the system failure [6-8].

In [9] analysis of compensation influence of the system input velocity on the lenght change of protector between two neighboring transporters is done. Also, the compensation influence of the system input velocity on velocities of all transporters is analyzed. In order to compensate the influence of the input velocity on the statical error, the potentiometer is inserted and it is the same for every cascade because the input velocity has the same influence on the statical error in every cascade.

In order to eliminate influence of protector compression coefficients another additional potentiometer must be inserted in each cascade. This is assured in our paper with simulation results using Matlab/Simulink [10].

\section{COMPENSATION OF PROTECTOR COMPRESSION COEFFICIENTS INFLUENCE}

Let us analyzed the cascade system shown in Fig.1, $[7,9]$. The structural scheme of this system with the compensation of protector compression coefficients influence is presented in Fig.2. 


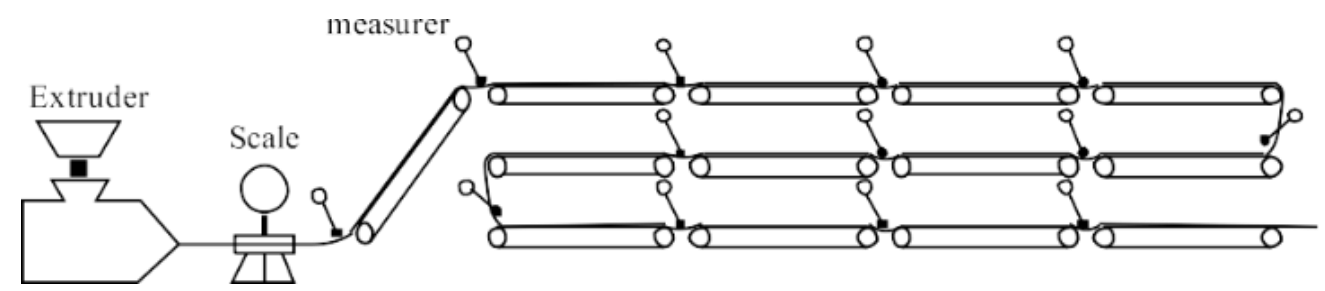

Fig. 1. Rubber strip transportation system

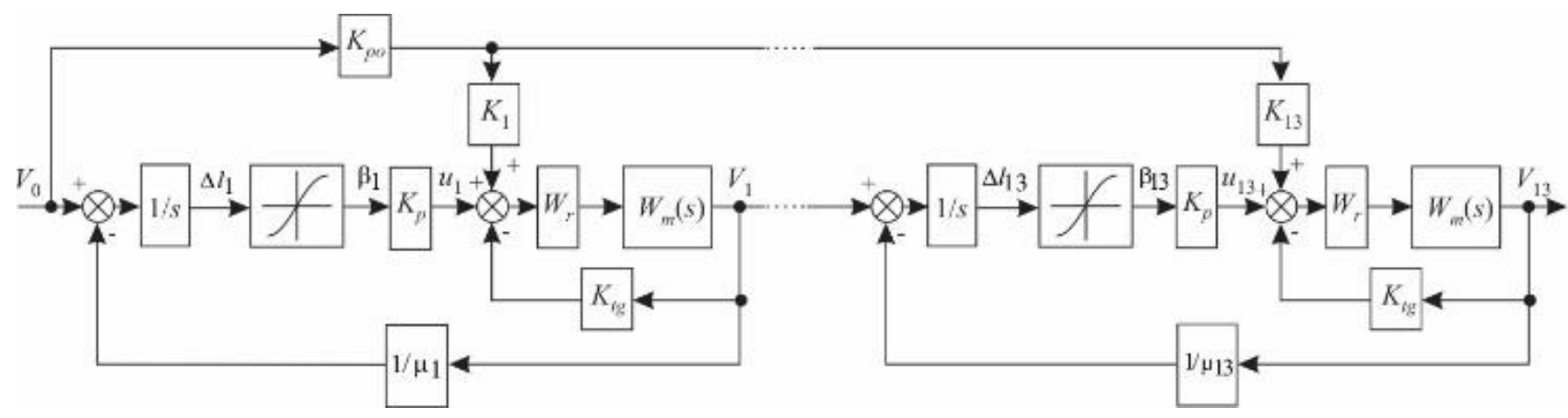

Fig. 2. The structural scheme of rubber strip transportation system with the compensation of protector compression coefficients influence

The following notations are introduced:

- $V_{o}$ is the input velocity,

- $l_{i}$ is the length of the rubber strip between the $i$-th and the $(i+1)$ - th transporters,

- $V_{g, i}^{(1)}$ is the velocity of the rubber strip at the beginning of the $i$-th transporter,

- $V_{g, i}^{(2)}$ is the velocity of the rubber strip at the end of the $i$-th transporter,

- $V_{i}$ is the velocity of the $i$-th transporter,

- $\mu_{i}$ is the rubber strip compression coefficient in the $i$-th transporter,

- $\beta_{i}=\Phi\left(\Delta l_{i}\right)$ is the nonlinear measurer characteristic,

- $W_{m}(s)=\frac{K_{m}}{T_{1} T_{2} s^{2}+\left(T_{1}+T_{2}\right) s+1}$ is the transfer function of the motor with the load,

- $T_{1}, T_{2}$ are the time constants of the subsystem loaded with rubber strip material,

- $K_{p o}$ is the additional gain for the elimination of the influence of the input velocity changes on $\Delta l_{i},[9]$,

- $\Delta l_{i}$ is the protector length change between two neighbor transporters,

- $W_{r}$ is the transfer function of thyristor regulator,

- $K_{r}$ is the thyristor regulator gain,
- $K_{t g}$ is the tahogenerator gain,

- $K_{p}$ is potentiometer gain,

- $K_{i}, i=1, \ldots, 13$ are potentiometer gains for the compensation of protector compression coefficients influence.

System dynamic is described by the following equations:

$$
\begin{gathered}
\frac{d l_{i}}{d t}=V_{g, i-1}^{(2)}-V_{g, i}^{(1)} \\
V_{g, i-1}^{(2)}=V_{i-1,} V_{g, i}^{(1)}=\frac{1}{\mu_{i}} V_{i}
\end{gathered}
$$

Relations (1) and (2) show that the rubber length between two transporters is equal to the integral of the difference between the velocity at the end of the former and the velocity at the beginning of the next transporter. From the relation (2) can be seen that the rubber strip velocity is equal to the velocity at the end of the transporter, while is not equal to the velocity at the beginning of the transporter. The velocity at the beginning of the transporter is $\mu_{i}$ times less than the transporter velocity.

Substituting relation (2) into relation (1) the next set of relations is obtained:

$$
\frac{d l_{i}}{d t}=V_{i-1}-\frac{1}{\mu_{i}} V_{i}
$$




$$
\Delta l_{i}=\frac{1}{s}\left(V_{i-1}-\frac{1}{\mu_{i}} V_{i}\right)
$$

The dependence of the measurer angles on rubber length changes is given by:

$$
\beta_{i}=\Phi\left(\Delta l_{i}\right)
$$

The goal is to keep $\Delta l$ at the constant value, and at zero in the case of the steady state. In the steady state the input value of the integrator, i.e., error, must be equal to zero. So:

$$
V_{i}=\mu_{i} V_{i-1}=\prod_{k=1}^{i} \mu_{k} V_{0}
$$

In real system [2,3], the rubber strip compression coefficients have values between 0.9 and 1 . It is possible to determined the values of rubber strip compression coefficients of each cascade using next relation:

$$
\mu_{i}=\frac{0.7+0.3 e^{-\frac{i}{2.6}}}{0.7+0.3 e^{-\frac{i-1}{2.6}}}, i=1, \cdots, 13
$$

Obtained values for $\mu_{i}$ are the theoretical values. One way for compensation of protector compression coefficients influence is to place one potentiometer $K_{i}, i=1, \cdots, 13$ in each cascade, Fig.2. Value of $K_{i}$ is adjusted such that $\Delta l_{i}$ has the same value in each cascade and approximately equal to zero. The transfer functions of motor, $W_{m}$, and thyristor regulator, $W_{r}$, in the steady state become gains, so that the following applies:

$$
s \rightarrow 0, W_{m}=K_{m}, W_{r}=K_{r}
$$

Using the structural scheme of rubber strip transportation system with the compensation of protector compression coefficients influence, Fig.2, next relations are obtained:

$$
\begin{gathered}
u_{i}=\left(\frac{1}{K_{r} K_{m}}+K_{t g}\right) V_{i}-V_{0} K_{p o} K_{i} \\
\Delta l_{i}=\Phi^{-1}\left[\frac{1}{K_{p}}\left(\frac{1}{K_{r} K_{m}}+K_{t g}\right) V_{i}-V_{0} \frac{K_{p o} K_{i}}{K_{p}}\right]
\end{gathered}
$$

Substituting equation (6) into (10) the next relation is obtained:

$\Delta l_{i}=\Phi^{-1}\left\{\frac{V_{0}}{K_{p}}\left[\left(\frac{1}{K_{r} K_{m}}+K_{t g}\right) \prod_{k=1}^{i} \mu_{k}-K_{p o} K_{i}\right]\right\}$

In order to $\Delta l_{i}$ become zero, from (11) can be concluded:

$$
\begin{gathered}
K_{i}=\prod_{k=1}^{i} \mu_{k} \\
K_{p o}=\frac{1}{K_{r} K_{m}}+K_{t g}
\end{gathered}
$$

In the next section the influence of gain $K_{i}, i=$ $1, \cdots, 13$, (12) on compensation of protector compression coefficients influence will be analyzed using program package Matlab/Simulink.

\section{MODELLING AND SIMULATION OF COMPENSATED SYSTEM USING MATLAB/SIMULINK}

Based on (7) and (12) values for $\mu_{i}$ and $K_{i}, i=$ $1, \cdots, 13$ are calculated for each cascade. These values are shown in Table 1.

Matlab/Simulink block diagram of rubber strip transportation system with the compensation of protector compression coefficients influence is shown in Fig.3. System parameters are $K_{t g}=$ $0.36, K_{r}=150, K_{m}=0.167$.

System input is ramp signal with initial velocity of $100 \mathrm{~mm} / \mathrm{s}$ and maximum velocity of $1500 \mathrm{~mm} / \mathrm{s}$ after $60 \mathrm{~s}$. This input signal is formed using block MATLAB Fcn in Fig. 3 and the next M-file:

\%function MATLAB FCN dr

function $y=d r(p)$

if $p<=60$, $y 1=100+(1400 * p) / 60$;

else if $p>60$,

$\mathrm{y} 1=1500$;

end;

end;

$y=y 1$;

Table 1. Values for $\mu_{i}$ and $K_{i}, i=1, \cdots, 13$ of each cascade

\begin{tabular}{|l|l|l|l|l|l|l|l|}
\hline & 1. & 2. & 3. & 4. & 5. & 6. & 7. \\
\hline$\mu_{i}$ & 0.9042 & 0.9279 & 0.9471 & 0.9620 & 0.9731 & 0.9812 & 0.9869 \\
\hline$K_{i}$ & 0.9042 & 0.8390 & 0.7946 & 0.7644 & 0.7438 & 0.7298 & 0.7202 \\
\hline & 8. & 9. & 10. & 11. & 12. & 13. & \multirow{2}{*}{} \\
\hline$\mu_{i}$ & 0.9910 & 0.9938 & 0.9958 & 0.9971 & 0.9980 & 0.9987 & \\
\cline { 1 - 5 }$K_{i}$ & 0.7137 & 0.7093 & 0.7063 & 0.7043 & 0.7029 & 0.7020 & \multicolumn{1}{|l}{} \\
\cline { 1 - 3 }
\end{tabular}




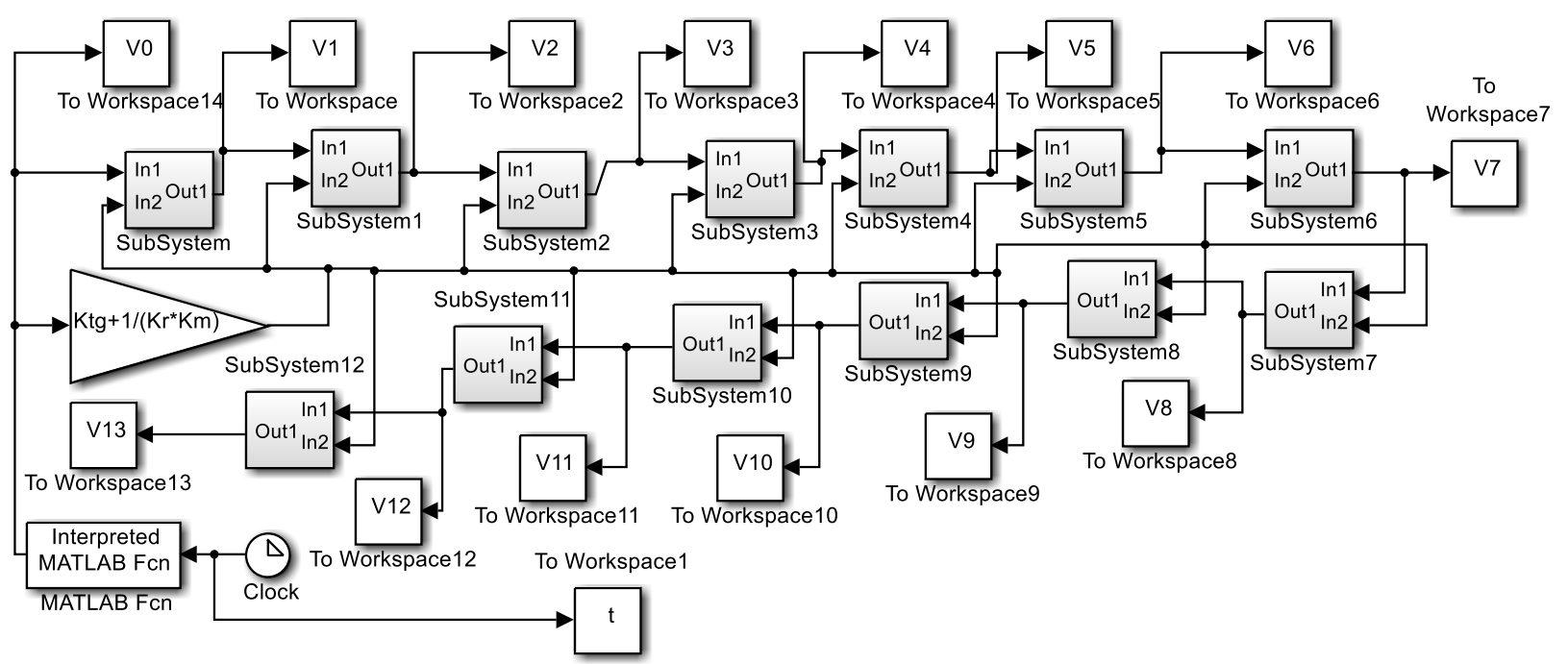

Fig. 3. Matlab/Simulink block diagram of rubber strip transportation system with the compensation of protector compression coefficients influence

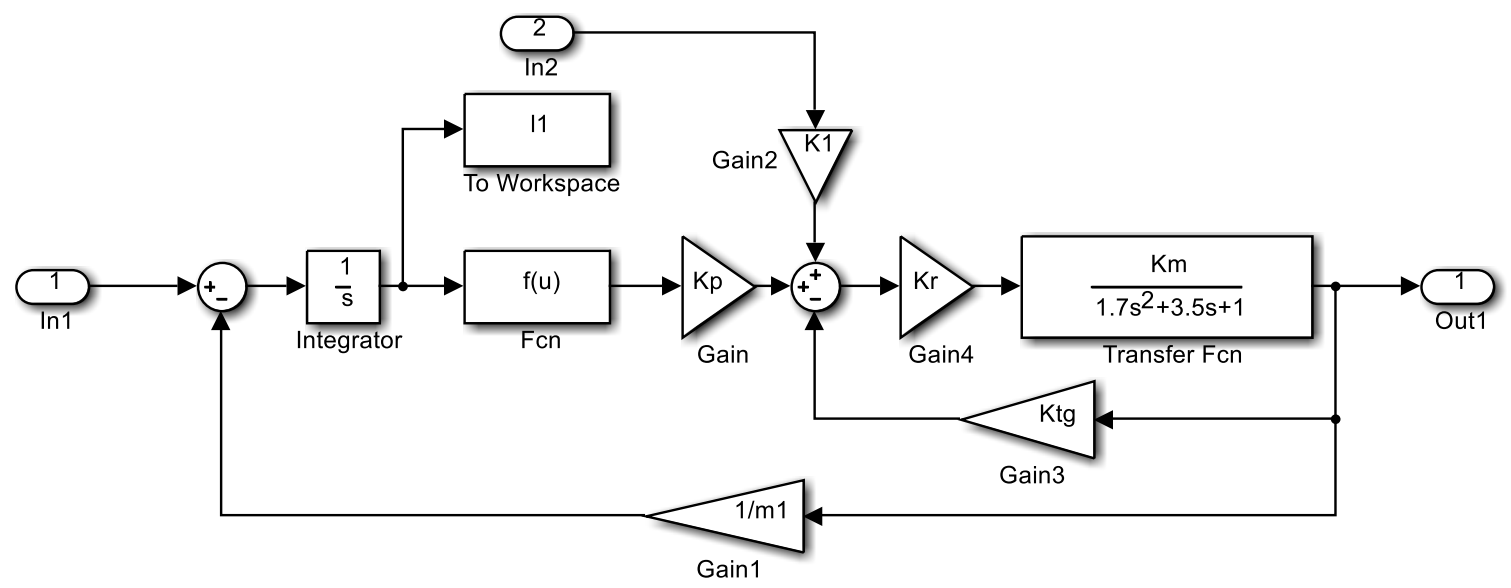

Fig. 4. Matlab/Simulink block diagram of SubSystem block from Fig.3

Each block SubSystem from Fig.3 represents one cascade of transportation system. Double click on this block, the block scheme of the entire cascade is opened. In Fig. 4 the block scheme of the first cascade is shown. The value of $K_{p}$ in block Gain is equal to 8 , and the values of $K_{1}$ and $\mu_{1}$ are equal to the values given in Table1. In Fig. 4 value of parameter $\mu_{1}$ is inserting in the block Gain1, with use of label $m_{1}$ instead of $\mu_{1}$.

The nonlinear measurer characteristic $\beta=$ $\Phi(\Delta l)$ is approximated by the next function:

$$
\beta=\operatorname{sign}(\Delta l) \frac{\pi}{6}\left(1-e^{-0.01|\Delta l|}\right)
$$

and shown in Fig.5. In Matlab/Simulink block diagram, Fig.4, nonlinearity (14) is implemented into the block Fcn, Fig.6.

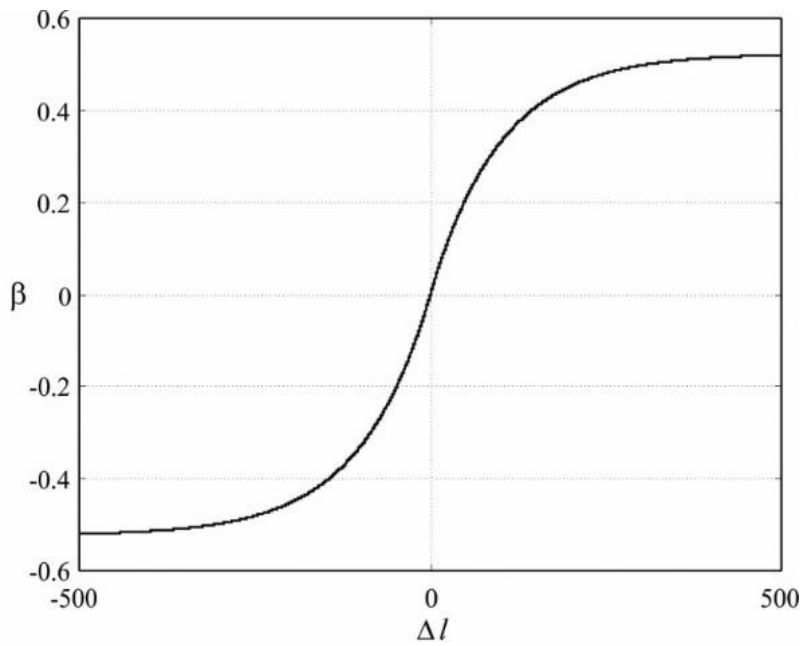

Fig. 5. Nonlinear characteristic $\beta=\Phi(\Delta l)$ 


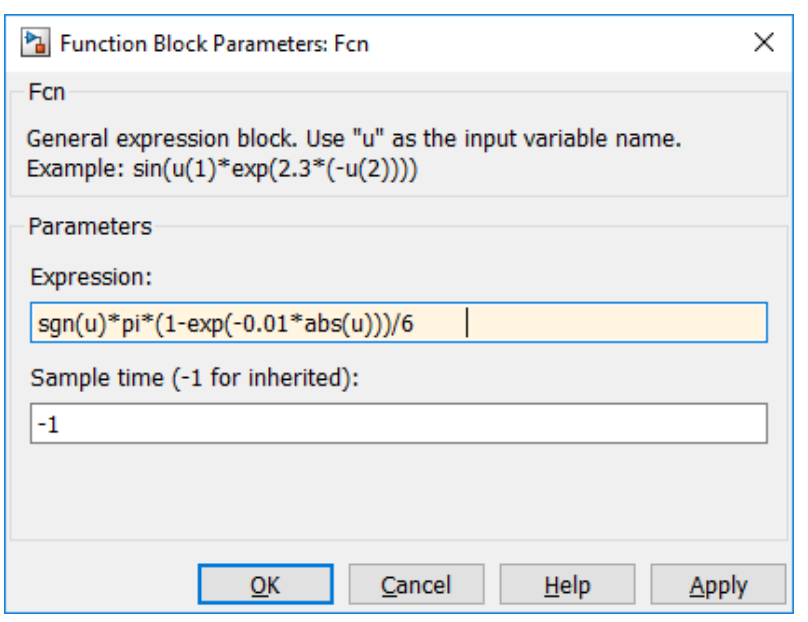

Fig. 6. Function block Fcn

Simulation result, i.e., time dependence of $\Delta \mathrm{l}_{\mathrm{i}}, \mathrm{i}=1, \cdots, 13$ in the case of compensation of protector compression coefficients influence is shown in Fig.7. It can be seen that after a while, $\Delta l_{i}$, has value equal to zero in each cascade. The highest peak occurs in the first cascade and with each cascade peak value, $\Delta l_{i}$, decreases. The peak $\Delta l_{i}$ does not occure in all cascades in the same time because transporters are in series connections and every transporter has its own peak delay. The first cascade is stabilized after $105 \mathrm{~s}$ and the last one after $300 \mathrm{~s}$. The maximum peak value, $\Delta l_{1}$, in the first cascade is $118 \mathrm{~mm}$ which is about half of the maximum permissible compression.

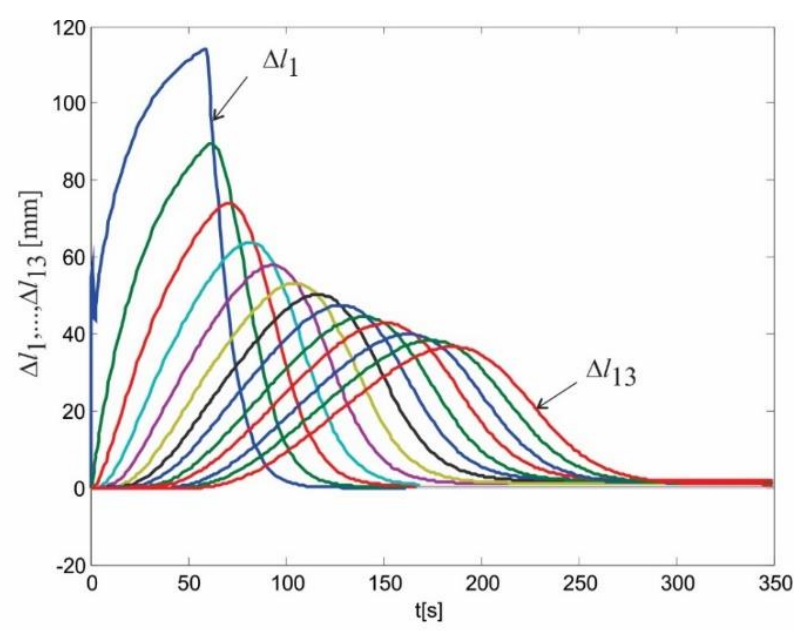

Fig. 7. Time dependence of $\Delta \mathrm{l}_{\mathrm{i}}, \mathrm{i}=1, \cdots, 13$ in the case of compensation of protector compression coefficients influence

In Fig.8 time dependences of all transporters velocities in the case of compensation of protector compression coefficients influence are shown. In the same figure the input velocity $V_{0}$ is shown, also. It can be seen that the velocities in the initial transporters are different. With increasing the ordinal number of cascade, velocities decrease. It may be noted that starting from the eighth cascade to the last thirteen, transporters valocities have approximately the same value as one might expect on the basis of parameter values shown in Table 1.

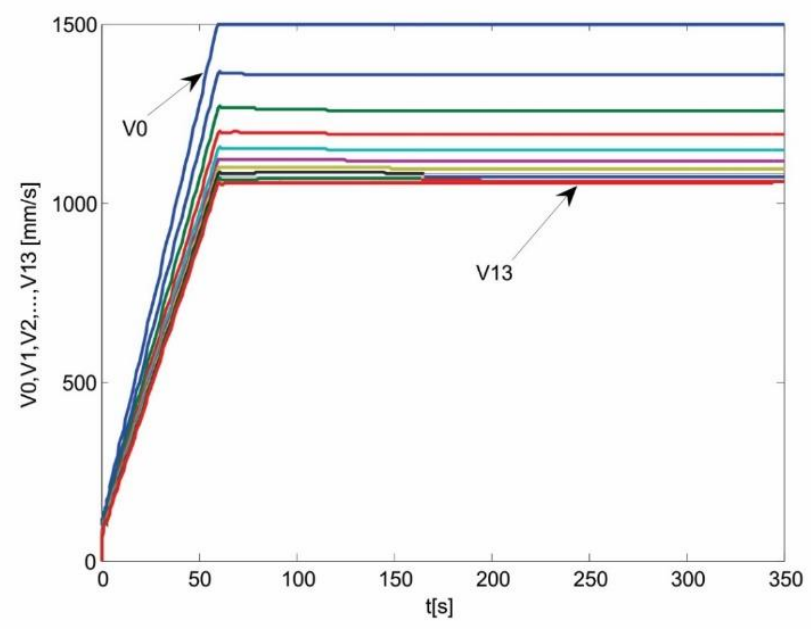

Fig. 8. Time dependences of all transporters velocities in the case of compensation of protector compression coefficients influence

\section{CONCLUSION}

System consisting of 13 cascades for protector cooling is presented in this paper. In this system the compensation of protector compression coefficients influence is performed for two cases:

1. protector lenght change between two neighbour transporters and

2. all transporters valocities.

In order to compensate the protector compression coefficients influence on static error, potentiometers $K_{i}, i=1, \ldots, 13$, different for each cascade are introduced into the system. This is confirmed by simulations using Matlab/Simulink.

\section{REFERENCES}

[1] И. В. Левинсон, Транспортние устројства непреривного дејствија. Масгиз, Москва, 1983. (In Russian)

[2] B. Danković, On the appearance of oscillations in cascade systems for transport of rubber strip materials, Proc. JUREMA 34, 1989, Vol.I, pp.97-100.

[3] B. Danković, Z. Jovanović, On oscillations in automatic controlled cascade systems for 
transport of rubber strip materials. Elektrotehnika, 43, 1994: E1-E3.

[4] B. Danković, M. Stanković, B. Vidojković, Simulation of convergent, oscillatory and chaotic dynamics of cascade systems, Proceedings of the seventh Symposium of Mathematics and its Applications, Timisoara, 1997, pp.101-106.

[5] B. Vidojković, B. Danković, Simulation of chaotic dynamic of cascade system using MATLAB, YU INFO 98, Kopaonik, Serbia, 1998, pp. 497-500.

[6] B. Danković, B. Vidojković, B. M. Vidojković, On the chaos in cascade systems for rubber strip transportation, Proceedings, The Fourth International Conference, Heavy Machinery, HM 2002, 2002, Kraljevo, Serbia, pp. A.97A.100.

[7] B. Samardžić, B. M. Zlatković, Simulation of bifurcation and escape-time diagrams of cascade-connected nonlinear systems for rubber strip transportation, Nonlinear Dynamics, 67 (2), 2012: 1105-1113.

https://doi.org/10.1007/s11071-011-0054-y

[8] B. M. Zlatković, B. Samardžić, On the spatial chaos appearance in cascade connected nonlinear transportation systems with trapezoidal nonlinearity transition, VIII International Conference Industrial Engineering and Environmental Protection (IIZS 2018), 11 - $12^{\text {th }}$ October 2018, Zrenjanin, Serbia, pp.147-154.

[9] B. M. Zlatković, B. Samardžić, Control analysis of the protector cooling system in tyre industry in program package MATLAB/SIMULINK, Continuous training, The basis for improving occupational safety, 15. International Conference, 2018, Kladovo, Serbia, pp.202-210.

[10] MATLAB Simulink-Tutorialspoint https://www.tutorialspoint.com>matlab 